\title{
Optimizing Energy Storage Participation in Emerging Power Markets
}

\author{
Hao Chen*, Zhenhua Liu ${ }^{\dagger}$, Ayse K. Coskun* and Adam Wierman ${ }^{\ddagger}$ \\ *Electrical and Computer Engineering, Boston University \\ ${ }^{\dagger}$ Applied Mathematics and Statistics, Stony Brook University \\ $\ddagger$ Computing and Mathematical Sciences, California Institute of Technology
}

\begin{abstract}
The growing amount of intermittent renewables in power generation creates challenges for real-time matching of supply and demand in the power grid. Emerging ancillary power markets provide new incentives to consumers (e.g., electrical vehicles, data centers, and others) to perform demand response to help stabilize the electricity grid. A promising class of potential demand response providers includes energy storage systems (ESSs). This paper evaluates the benefits of using various types of novel ESS technologies for a variety of emerging smart grid demand response programs, such as regulation services reserves (RSRs), contingency reserves, and peak shaving. We model, formulate and solve optimization problems to maximize the net profit of ESSs in providing each demand response. Our solution selects the optimal power and energy capacities of the ESS, determines the optimal reserve value to provide as well as the ESS real-time operational policy for program participation. Our results highlight that applying ultra-capacitors and flywheels in RSR has the potential to be up to 30 times more profitable than using common battery technologies such as LI and LA batteries for peak shaving.
\end{abstract}

\section{INTRODUCTION}

A sustainable energy future mandates integrating a larger portion of renewable generation into the grid. Most states in the US and several European countries already have aggressive targets to increase the share of renewables in their portfolios [1], [2]. The fact that many forms of renewable generation are intermittent by nature (e.g., wind and solar) creates significant challenges for grid operators, who need to match supply and demand in real-time. In response to this challenge, emerging ancillary power markets provide sizable monetary incentives for the consumers to perform demand response, which refers to a consumer adjusting its own electricity use following a set of constraints or directives given by the grid operator.

Among potential demand response program participants, data centers, electrical vehicles (EVs), and smart buildings are especially promising, and have received recent attention from the research community [3], [4], [5], [6]. This attention is due to their significant flexibility in energy consumption, as well as the large cumulative power consumption levels and/or fast growth these entities provide.

One of the most promising participation opportunities for demand response comes from using energy storage systems (ESSs), which can potentially charge/discharge depending on the demand response program requirements reliably. There are a variety of energy storage startup companies [7], [8] that use ESSs to participate directly in energy market programs this way. Additionally, entities such as data centers and smart buildings, which have on-site ESSs to manage power outages, can make use of ESSs to receive monetary incentives without having to alter their internal performance. ESSs have been studied for participation in well-known power programs such as real-time pricing [9], but the potential of ESS participation in many of the most promising demand response programs has yet to be understood, including regulation service reserves (RSR), contingency reserves in emerging ancillary service markets, and peak shaving programs. Some recent work has begun to investigate these programs or ESS capacity planning [10], [11], [12], [13]; however, in most cases, these papers use simplified participation models, e.g., an RSR model that ignores regulation accuracy constraints and penalties [14]. Besides, few work studies the decisions of reserve value and the ESS capacity planning. Furthermore, different ESS technologies (e.g., lead-acid (LA) batteries, lithium-ion (LI) batteries, ultra/super-capacitors (UC), flywheels (FW), and compressed air energy storage (CAES)) have contrasting properties, which can dramatically impact profits of participation in such programs. Systematic evaluation and comparison of the benefits of using these ESS technologies in a variety of demand response opportunities do not exist in current literature.

This paper's goal is to thoroughly evaluate, optimize, and contrast a range of ESS technologies for participation in a variety of promising demand response programs. Our method seeks to provide a strategy for the selection and management of ESSs for a broad range of consumers (data centers, EVs, smart buildings) to maximize the incentives received from ancillary power markets, and hence, to minimize the electricity cost while helping stabilize the grid. Our specific contributions are:

First, we provide detailed models and optimization solutions for participation of ESSs in multiple smart grid programs, including RSR, contingency reserves and peak shaving (Sections $\Pi$ and $\Pi$. We also design practical heuristic solutions that handle the real life probabilistic constraints in RSR provisioning problem (Section IV-A). In each model, the cost of ESS equipment, the revenue received for demand response, and constraints required by the demand response program are formulated. The net profits are optimized based on these models, and the corresponding optimal decisions of reserve value, ESS capacity planning and the operational policies are derived. The generality and wide applicability of the models and solutions distinguish this paper from previous work.

Second, the proposed models and optimal solutions enable, for the first time in the literature, a thorough comparison of the benefits of different ESSs for participation in demand response opportunities (Section III). We highlight the ESS technology 
that is the most appropriate for each power program (and viceversa). Results show that UC is the most profitable ESS for RSR, while LI battery is the best choice for peak shaving. Also, we show that none of today's typical ESSs can earn positive net profits from providing contingency reserves.

Finally, in addition to evaluation with offline optimization solutions, this paper proposes heuristic practical online policies for provisioning with different types of ESSs in RSR program (Section IV-B), which is the most profitable program among those studied. As opposed to the offline solution, our online solution does not require information of RSR signal in advance, and thus, is applicable for real-life use. The solution adaptively leverages the tolerable RSR signal tracking errors for pursing larger profits. Our solution is able to satisfy all constraints and thus guarantee the feasibility of the provision, while still achieving significant profits.

\section{ENERGy StORAGE SyStems}

Storage technologies are becoming more cost-effective and wide spread and, at the same time, more lucrative market participation opportunities are emerging. In this section, we provide an overview of some potential storage technologies and define a model that enables us to study the participation of each ESS in various market opportunities.

\section{A. Background on Energy Storage Systems}

In this paper, we focus on five popular ESSs, namely, lead-acid (LA) batteries, lithium-ion (LI) batteries, ultra/supercapacitors (UC), flywheels (FW), and compressed air energy storage (CAES). In the following, we briefly highlight important characteristics of each. The interested reader can refer to prior work [15] for more information.

Lead-Acid (LA) batteries are widely used in daily life, e.g., in car batteries. They have very low self-discharge loss rates, which makes them suitable for the demand response programs with long durations, e.g., hours. Additionally, they have moderate energy cost and power cost, and therefore are robust under different market scenarios. However, the key disadvantage of LA batteries is the relatively small number of charge/discharge cycles and shorter float life. LA batteries can only be used for several thousand circles.

Lithium-Ion (LI) batteries are also widely used in our daily life, and have similar characteristics to LA batteries. The key difference is that LI batteries have relatively higher costs, longer lifetimes, more cycles, and higher efficiency.

Ultra/super-Capacitors (UCs) differ dramatically from LI and LA batteries. UCs have an extremely high tolerance for frequent charging/discharging. Additionally, UCs have high efficiency and power density. However, they have a high energy cost (around $\$ 10,000 / \mathrm{kWh}$ ) and high self-discharge rate.

Flywheels (FWs) represent a middle ground between LI/LA batteries and UCs. Like UCs, they have high efficiency and power density, but also high energy cost and a high selfdischarge rate.

Compressed Air Energy Storage (CAES) has a very low energy cost and self-discharge rate. However, it has a very slow ramping time (10 min vs. $1 \mathrm{~ms}$ in the other four ESSs). This means that it cannot adapt quickly, which limits participation
TABLE I. A SELECTION OF TODAY'S TYPICAL CAPACITIES OF ESSS, BASED ON SPACE CONSTRAINTS.

\begin{tabular}{|c|c|c|c|c|c|}
\hline & LA & LI & UC & FW & CAES \\
\hline$P_{c a p}(\mathrm{~kW})$ & 1,000 & 1,000 & 20,000 & 10,000 & 20 \\
\hline$E_{c a p}(\mathrm{kWh})$ & 250 & 250 & 250 & 250 & 250 \\
\hline
\end{tabular}

of CAES in some market programs. Additionally, it has a very low energy density (large space needed) and a high power cost.

\section{B. Modeling Energy Storage Systems}

There are two key components in modeling ESSs: costs (both of procurement and operation) and operation constraints (self-discharging, ramping, etc.). Operation constraints can be classified into (i) constraints imposed by the ESS technology and (ii) constraints imposed by the demand response program. Constraints of type (i) are discussed here, and constraints of type (ii) are discussed in Section 【II

ESS Costs: The life span of an ESS is normally years with one-time upfront purchase/installation cost, yet participation in a demand response program can span a year, a month, or even a day. In order to handle the mismatch in time granularity, we amortize the upfront cost evenly over the lifespan of the ESS. Let $P_{\text {cap }}$ (in $\mathrm{kW}$ ) and $E_{\text {cap }}$ (in $\mathrm{kWh}$ ) represent the power capacity and energy capacity of the ESS, respectively, and $\Pi^{P}$ (in $\$ / \mathrm{kW}$ ) and $\Pi^{E}$ (in $\$ / \mathrm{kWh}$ ) are the corresponding prices. Then the one-time upfront cost is 1 :

$$
\Pi^{P} P_{c a p}+\Pi^{E} E_{\text {cap }} .
$$

Two factors that need to be considered to calculate the duration of use are the face-plate lifetime $T_{\max }$ and the maximal number of charge/discharge cycles $L_{c y c}$. Assuming the charge/discharge frequency is $f_{j}$, the effective duration of use is $\min \left\{T_{\max }, \frac{L_{c y c}}{f_{j}}\right\}$. Since many of the demand response programs clear the credits daily, we amortize the cost of ESSs into daily prices, namely, for each type of ESS $k$, we define its daily power and energy capacity prices as $\Pi_{k}^{P, d}$ and $\Pi_{k}^{E, d}$ as:

$$
\Pi_{k}^{P, d}=\frac{\Pi_{k}^{P}}{\min \left\{T_{\max }, \frac{L_{c y c}}{f_{j}}\right\}}, \quad \Pi_{k}^{E, d}=\frac{\Pi_{k}^{E}}{\min \left\{T_{\max }, \frac{L_{c y c}}{f_{j}}\right\}},
$$

where $f_{j}$ is the frequency of the charge/discharge in program $j$. Therefore, the daily amortized cost is:

$$
\Pi_{k}^{P, d} P_{c a p}+\Pi_{k}^{E, d} E_{c a p} .
$$

ESS Operation Constraints: Assume that at time $t$, the charge and discharge rates of an ESS are $r_{t}$ and $d_{t}$, respectively. We denote the total energy stored in the ESS at time $t$ as $e_{t}$, and the overall power rate from the view of the system level as $u_{t}$. Then we have:

$$
\begin{array}{r}
e_{t}=e_{t-1}-\mu e_{t-1}+r_{t}-d_{t}, \forall t, \\
u_{t}=r_{t} / \eta-d_{t}, \forall t,
\end{array}
$$

where $\mu$ is the self-discharge rate of the ESS, and $\eta$ is the energy charging efficiency. We have $\eta<1$, as there is always amount of loss during the ESS charge process. $\mu$ and $\eta$ vary

\footnotetext{
${ }^{1}$ Other ways of calculating the upfront cost exist (e.g., the upfront cost is selected as the maximum of the costs on power capacity and energy capacity [15]). Our method is adaptable to such calculations, e.g., an ancillary variable can be introduced to convert the selection of the maximum on power and energy capacities into two linear constraints.
} 
with types of ESSs. For example, UC and FW in general have higher efficiency than LA and LI batteries, however, they have much higher self-discharge rate.

The charge and discharge rates are also constrained by the charge/discharge capacities of the ESS, as follows:

$$
0 \leq r_{t} \leq \frac{P_{c a p}}{\gamma}, 0 \leq d_{t} \leq P_{c a p}, \forall t,
$$

where $P_{c a p}$ is the power capacity of the ESS defined before, $\gamma$ is the ratio of discharge rate to charge rate. For $\mathrm{UC}$ and $\mathrm{FW}, \gamma$ is close to 1 , which means they have almost same charge and discharge capacities, however for LA and LI batteries, $\gamma>1$, representing a (much) slower recharge rate.

The amount of energy that is stored in the ESS is constrained by the ESS energy capacity $E_{c a p}$. In addition, it is constrained by the Depth of Discharge (DoD), which helps guarantee the lifetime of the equipment:

$$
(1-D o D) E_{c a p} \leq e_{t} \leq E_{c a p}, \forall t .
$$

Finally, though most ESSs are able to ramp up their discharge rate extremely fast, some ESSs, e.g., CAES, cannot. Thus, we have the discharge rate ramp up constraint:

$$
d_{t+1}-d_{t} \leq \frac{P_{c a p}}{T^{r a m p}}, \forall t,
$$

where $T^{r a m p}$ is the time for ESS to ramp up the discharge rate from 0 to $P_{\text {cap }}$.

\section{MARKET OPPORTUNITIES FOR ENERGY STORAGE SYSTEMS}

In this section, we propose detailed models of ESS participation in various electricity market programs, including RSR, contingency reserves, and peak shaving. We introduce the revenue function, Revenue ${ }_{j}$ that represents the revenue received from participation in the program $j$, and the constraints, Constraint $_{j}$ that are required by the program operator. The net profit of participation equals to Revenue ${ }_{j}$ minus the daily amortized cost of ESS in Eq. (3). For each type of ESS $k$ and each program $j$, we derive the optimal selections of ESS energy and power capacities, as well as the optimal ESS operational policy (including the amount of reserves to provide, and the solution of how to dynamically charge and discharge over time, etc.) for maximizing profit. Then we evaluate applying these ESSs with today's typical capacities, and conduct sensitivity analysis of the maximal net profit on the price of reserves. Finally, we compare the benefits of these ESSs participating in each program.

\section{A. Regulation Service Reserves (RSR)}

Historically, RSRs were mainly provided by centralized generators, but market rules are changing to encourage demand-side participation. This emerging demand response opportunity is quite attractive due to the high payments comparable to the real-time market price [16], [17]. RSR programs are typically quite demanding for participants. Each RSR provider is obligated to modulate its power to track an RSR signal $\beta_{t}$ broadcast every 4 seconds (this defines the length of one time slot) by the independent system operator (ISO) [16]. The signal is between $[-1,1]$, with an average of zero over long time intervals. It is updated every 4 seconds in increments that do not exceed $\pm 4 / \tau$, where $\tau$ is in 100-300 seconds [18].

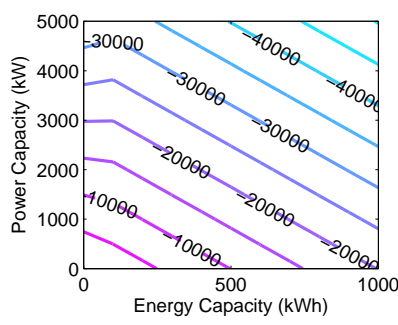

(a) Profit of LI batteries (\$/day).

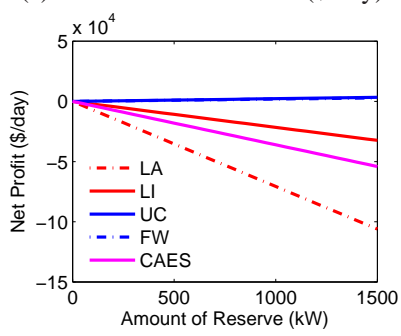

(c) Impacts of $R$ on net profit.

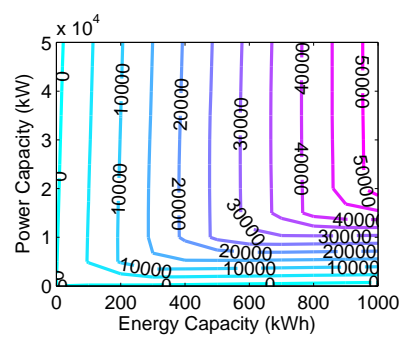

(b) Profit of UC (\$/day).

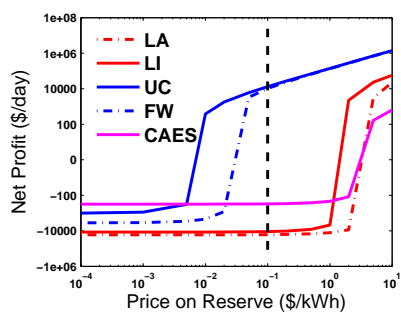

(d) Impacts of price on net profit
Fig. 1. ESSs in regulation service reserves.

1) Problem Formulation: A provider receives $\Pi^{R S} \cdot R$ revenue for providing $R(\mathrm{~kW})$ amount of reserves, where $\Pi^{R S}$ is the price of reserves. The revenue is reduced based on the tracking error of the RSR signal, i.e., $\left|u_{t}-R \beta_{t}\right|$, where $u_{t}$ is the power rate defined in Eq.(4). The overall daily revenue received from RSR participation ( $T=1$ day) is:

$$
\text { Revenue }_{R S}=\Pi^{R S} R-\theta \cdot \Pi^{R S}\left(\frac{1}{T} \sum_{t=1}^{T}\left|u_{t}-R \beta_{t}\right|\right),
$$

where $\theta$ is the penalty coefficient on the tracking error.

The provider may lose the RSR contract if the constraint on signal tracking performance is violated. We formulate this using a probabilistic constraint:

$$
\sum_{t=1}^{T} \mathbb{I}_{\left\{\left|\frac{u_{t}}{R \beta_{t}}-1\right| \leq \rho_{1}\right\}} \geq \rho_{2} T
$$

where $\rho_{1}$ and $\rho_{2}$ are parameters set by the ISO. This equation shows that the probability of tracking error at each time $t$, (i.e., $\left.\left|u_{t}-R \beta_{t}\right|\right)$ that is smaller than $\rho_{1} R\left|\beta_{t}\right|$ should be greater than or equal to $\rho_{2}$.

Putting Eq. (2) - Eq. (9) together, the overall optimization formulation of ESSs in RSR is:

$$
\begin{array}{r}
\underset{E_{c a p}, P_{\text {cap }}, R, \mathbf{r}, \mathbf{d}, \mathbf{u}, \mathbf{e}}{\max } \Pi^{R S} R-\theta \cdot \Pi^{R S} \frac{1}{T} \sum_{t=1}^{T}\left|u_{t}-R \beta_{t}\right| \\
-\left(\Pi^{P, d} P_{c a p}+\Pi^{E, d} E_{c a p}\right), \\
\text { s.t. } \quad \sum_{t=1}^{T} \mathbb{I}_{\left\{\left|\frac{u_{t}}{R \beta_{t}}-1\right| \leq \rho_{1}\right\}} \geq \rho_{2} T, \\
e_{t}=e_{t-1}-\mu e_{t-1}+r_{t}-d_{t}, \forall t \in[1, T], \\
u_{t}=r_{t} / \eta-d_{t}, \forall t \in[1, T], \\
0 \leq r_{t} \leq \frac{P_{c a p}}{\gamma}, 0 \leq d_{t} \leq P_{c a p}, \forall t \in[1, T], \\
(1-D o D) E_{c a p} \leq e_{t} \leq E_{\text {cap }}, \forall t \in[0, T], \\
d_{t+1}-d_{t} \leq \frac{P_{\text {cap }}}{T^{\text {ramp }}}, \forall t \in[1, T-1], \\
P_{\text {cap }} \geq 0, E_{\text {cap }} \geq 0, R \geq 0 .
\end{array}
$$

In the formulation we use $\mathbf{r}, \mathbf{d}, \mathbf{u}$ and $\mathbf{e}$ to denote the vectors of $r_{t}, d_{t}, u_{t}$ and $e_{t}$, respectively. The objective function is to maximize the net profit of the participation, recalling that the 
net profit equals the revenue for providing reserves (reduced by the tracking error) minus the amortized cost of ESS equipment. The constraints are imposed by both the demand response program (RSR here) and the ESS technology. The decision variables of this optimization problem are:

- $\quad$ Power and energy capacities of ESS, i.e., $\left(P_{c a p}, E_{c a p}\right)$;

- The amount of reserve to provide, i.e., $R$;

- $\quad \mathbf{r}, \mathbf{d}, \mathbf{u}$ and $\mathbf{e}$, which represent how the ESS is operated dynamically, i.e., the operational policy.

2) Case Study: To evaluate the potential value from RSR program, we solve the above optimization formulation for the types of ESSs introduced before. We use parameters defined by prior work [15]. The RSR signal $\beta_{t}$ that we use is a real 24-hour signal from PJM [16]. Additionally, $\rho_{1}=0.2, \theta=1$ and $\Pi^{R S}=\$ 0.1 / \mathrm{kWh}$ based on today's markets [17].

The probabilistic constraint makes Eq. (10) not straightforward to solve. To simplify the problem, we first study the case of $\rho_{2}=1$, in which the probabilistic constraint in Eq.91 can be transformed to a deterministic constraint:

$$
\left|\frac{u_{t}}{R \beta_{t}}-1\right| \leq \rho_{1}, \forall t \in[1, T] .
$$

Heuristic solutions of $\rho_{2}<1$ will be discussed in Section IV Finally, the absolute value on the tracking error in Eq.(10) and Eq.(11) leads to piecewise linear property. We simplify the piecewise linear formulation to a linear one by introducing ancillary variables.

At the current reserve prices $\left(\Pi^{R S}=\$ 0.1 / \mathrm{kWh}\right)$, the optimal solution of Eq.101 for LA, LI batteries and CAES are all $P_{c a p}^{*}=E_{c a p}^{*}=R^{*}=0$, which demonstrates that there is no net profit of LA, LI batteries or CAES to participate in RSR program, i.e., the ESS cost of them is always larger than the revenue received from the program, no matter what the power and energy capacities are used or how they are operated dynamically. On the other hand, there is no feasible optimal solution of Eq. 10 for UC and FW: the net profit keeps increasing as $P_{c a p}, E_{c a p}$ and $R$ increase, which demonstrates that the maximal net profit is large for $\mathrm{UC}$ and $\mathrm{FW}$, as long as sufficiently large power and energy capacities can be offered. This highlights that the revenue earned by UC and FW from RSR is always larger than the amortized cost of the ESS.

We then study the sensitivity of net profit to energy, power capacities and the amount of reserve provision. Fig 1(a) and Fig 1(b) present the optimal net profit (the negative value represents that the cost of ESS is larger than the revenue, hence the net profit is less than 0) for varying energy and power capacities $\left(E_{c a p}, P_{c a p}\right)$, and for LI batteries and UC respectively, in contour plots. LA batteries have similar results to LI batteries, and FW is similar to UC. From the figures, we see that for LA/LI batteries, the net profits of participating RSR are always negative, and the larger capacities of them are used, the higher cost there would be. On contrary, for UC and FW, a larger $\left(E_{c a p}, P_{c a p}\right)$ creates larger net profit. The optimal net profit via varying amount of reserve, i.e., $R$, is shown in Fig 1(c), The net profits of LA, LI batteries and CAES are always negative and monotonously decrease along the increase of $R$, while the net profits of UC and FW are always larger than 0 and monotonously increase. Note that for all ESSs, providing larger $R$ requires larger ESS capacities.
The main factors that lead to such differences among ESSs are related to the characteristics of the ESSs. Since the RSR signal changes rapidly (every 4 seconds) and bidirectionally, in order to track it, RSR providers must have a large power capacity and large charge/discharge cycles. A large energy capacity, however, is not necessary, as the RSR signal has an average of zero over longer time intervals. UC and FW perfectly match these RSR characteristics: they have extremely high tolerance for frequent charging/discharging, high efficiency and power density, and relatively low power capacity cost, whereas under the high charge/discharge frequency in RSR, the lifetime of LA or LI batteries is shortened to less than 10 days due to the limited life cycle, which results in great cost and thus they no longer gain any net profit from RSR participation. CAES is even more limited due to the very large ramp up delay in discharge and the extremely small power density.

Next we focus on the RSR participation of different ESS technologies with today's typical capacities. In practice, the power and energy capacities of ESSs usually have upper bound limitations due to the restrictions of manufacturing techniques, unit prices and space constraints. Table I lists a selection of today's typical capacities of different types of ESSs referring to recent work [15], [19], [20], [21], estimated mainly based on space constraint:2. The power capacity of CAES is small due to its extremely small power density. The optimal net profit and the corresponding optimal $R^{*}$ of these typical ESSs in RSR are listed in the $3^{\text {rd }}$ row of Table II From the table, today's typical UC or FW can provide around 6MW RSR, and gain more than $\$ 10,000$ net profit a day, which are close to the power consumption and the cost of a data center with 10,00020,000 servers. The cost of this typical UC or FW is around $\$ 4$ million, which can be paid back in less than one year by receiving RSR credit.

Fig. 1(d) shows the optimal net profit via varying reserve price $\Pi^{R S}$, for different types of ESSs with their capacities fixed and given in Table I] The black dashed line represents where the current market reserve price is around. From the figure, LI, LA batteries and CAES start to gain net profit (the value of the net profit is larger than 0 ) when the reserve price $\Pi^{R S}$ is beyond $\$ 1 / \mathrm{kWh}$.

\section{B. Contingency Reserves}

In ancillary markets, contingency reserves are used to respond to loss of power supplies during generation or line failures. They are typically called by the market less than once a day, and some of them are called even less than once a year. A call typically lasts from several minutes to a few hours. Reserves that are able to respond immediately are known as spinning reserves, whereas reserves that require more time to respond are called non-spinning reserves. For example, NYISO provides 10-minute spinning and 10-minute non-spinning reserves. Another type of reserves, the operating reserves, are also provided by NYISO, as supplements of other reserves. Operating reserves have longer reaction time but also last longer, e.g., more than 30 minutes [17]. 10-minute spinning reserves have the highest price while the price of

\footnotetext{
${ }^{2}$ Since we have taken the cost and unit price information into account in the problem formulation, we no longer consider it as a problem in determining typical capacities of ESSs here.

${ }^{3}$ All results listed in Table $\amalg$ are the optimization solutions of Eq. 10 when $E_{c a p}$ and $P_{\text {cap }}$ are given as in Table [
} 


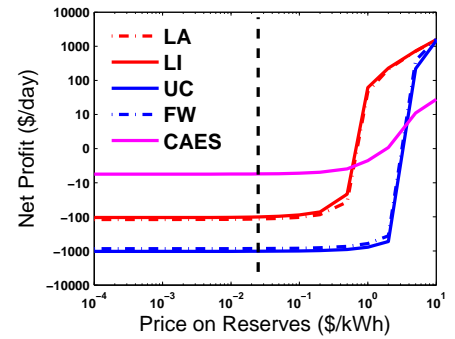

Fig. 2. The optimal net profit via varying contingency reserve prices $\Pi^{C R}$ for ESSs with today's typical capacities. The black dashed line shows where the current reserve price is at.

30-minute operating reserves is the lowest. All these prices are significantly lower than that of RSR. Overall, due to the much lower frequency of calls as well as the lower price of the reserves, the revenue received from contingency reserve provision is much lower than revenue from RSR provision.

1) Problem Formulation: The revenue of contingency reserves can be modeled as:

$$
\text { Revenue }_{C R}=\Pi^{C R} R,
$$

where $R$ is the amount of contingency reserves provided and $\Pi^{C R}$ is the price of the reserve. Unlike RSR, the contingency reserve provision is single directional with:

$$
r_{t}=0, \quad d_{t}=R, \forall t \in\left[T_{S}, T_{E}\right],
$$

where $\left[T_{S}, T_{E}\right]$ is a subset of $[1, T]$, representing that only at some $t$ during a day, an ESS is used to provide contingency reserves. For the rest of the day, the ESS is not used. When providing contingency reserve, the ESS keeps discharging at the fixed rate as the reserve value $R$. In order to provide the maximal amount of reserves, an ESS is charged to its full energy capacity before response, i.e.,

$$
e_{T_{S}}=E_{c a p} .
$$

We formulate the optimization problem for ESS in contingency reserves by putting Eq.(2)-(7) together with Eq.(12)(14). The objective function is still to maximize the net profit. The decision variables are the same as those of RSR.

2) Case Study: We focus on the 10-minute spinning reserve as an example of contingency reserves, as it is expected to have the highest revenue. $\Pi^{C R}=\$ 0.025 / \mathrm{kW}$ is selected for the 10-minute spinning reserve based on today's market information [17]. We assume the 10-minute spinning reserve is called once a day in our case, and $T_{E}-T_{S}=10 \mathrm{~min}$.

The optimal solution for all five ESSs in contingency reserve are: $P_{c a p}^{*}=E_{c a p}^{*}=R^{*}=0$, which shows that none of five ESSs gain net profit by only providing contingency reserves at today's market reserve price, no matter what the power and energy capacities are used, and how they are operated. The larger the capacities $\left(E_{c a p}, P_{c a p}\right)$ are used, the more reserves $R$ that an ESS can provide, however, as well as the higher the cost of ESS would be, and the cost is always larger than the revenue from providing $R$.

The $4^{\text {th }}$ row in Table III shows results of maximal net profit of contingency reserve and corresponding amount of reserve for today's typical ESS capacities, i.e., $\left(E_{c a p}, P_{c a p}\right)$ given from Table [] It highlights that none of today's typical ESSs earn profit from contingency reserves at today's reserve prices. Contingency reserves are demanding in terms of energy capacity (as opposed to power capacity), though the power

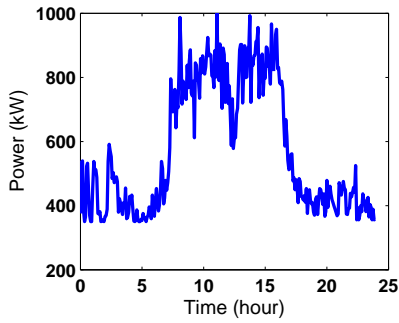

(a) 1-day power trace.

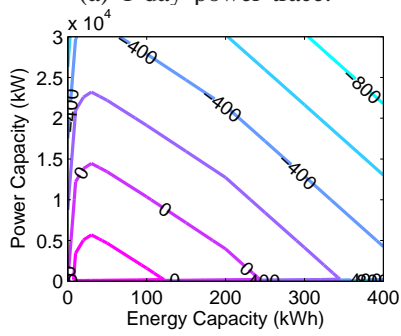

(c) Profit of UC (\$/day).

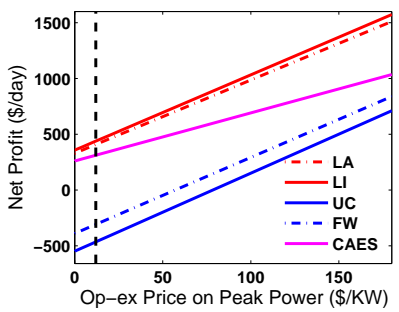

(e) Impact of op-ex price on profit.

Fig. 3. ESSs in peak shaving.

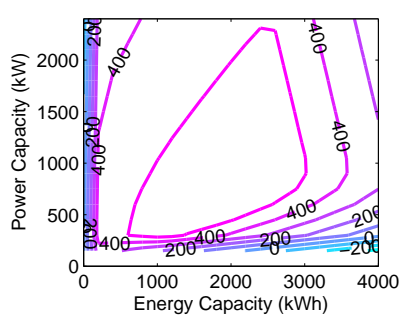

(b) Profit of LI batteries (\$/day).

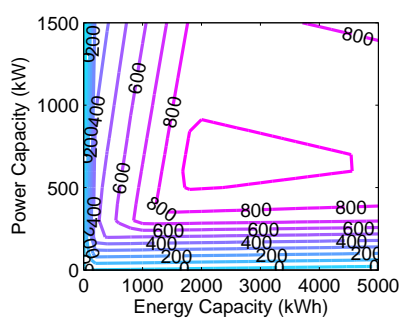

(d) Profit of CAES (\$/day).

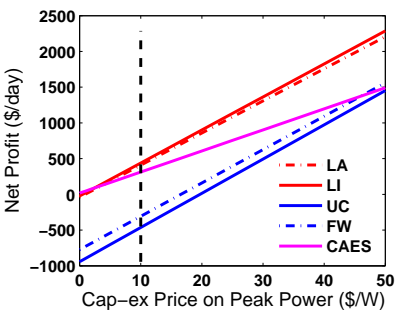

(f) Impact of cap-ex price on profit. capacity cannot be too low either. From the table, LA and LI batteries perform better than UC and FW, because of their lower price on energy capacity and relatively low selfdischarge rate, but still not well enough to be profitable. Fig 2 presents the optimal net profit via varying reserve prices $\Pi^{C R}$ for different ESSs. LI and LA batteries start to gain profit when the price is close to $\$ 1 / \mathrm{kWh}$, whereas the critical points of CAES, UC and FW are around $\$ 5-8 / \mathrm{kWh}$.

\section{Peak Shaving}

The electricity bill charged monthly by utilities to large commercial and industrial power consumers, i.e., the operational expenditure (op-ex), typically consists of two parts: (i) the energy charge and (ii) the charge for the peak power during the month. The peak power is the maximum in the month of average power over each 15-30 minute duration. The price of the peak power (i.e., the op-ex peak power price) is around $\$ 12 / \mathrm{kW} /$ Month currently. The one-time cost of building power infrastructure to provide capacities to satisfy the peak power requirements, i.e., the capital expenditure (cap-ex), is around $\$ 10-20 / \mathrm{W}$ on peak power based on current estimates [15]. Thus, cutting peak power is an important way to reduce costs. This approach, termed peak shaving, is common and ESS provides a key method for implementation.

1) Problem Formulation: When participating in peak shaving, an ESS that shaves $R$ amount of power from the peak power can gain revenue:

$$
\text { Revenue }_{P S}=\Pi^{P S} R,
$$

where $\Pi^{P S}$ is the overall price on shaved power, i.e., the summation of the amortized capital (cap-ex) price and operational 
TABLE II. OPtimal SOLUTIONS FOR PEAK SHAVING.

\begin{tabular}{|c|c|c|c|c|c|}
\hline & LA & LI & UC & FW & CAES \\
\hline$P_{c a p}^{*}(\mathrm{~kW})$ & $1.30 * 10^{3}$ & 769.19 & 148.39 & 147.85 & 645.36 \\
\hline$E_{c a p}^{*}(\mathrm{kWh})$ & $2.15 * 10^{3}$ & $2.40 * 10^{3}$ & 29.82 & 29.93 & $1.83 * 10^{3}$ \\
\hline Profit $(\$ /$ day $)$ & 607.40 & 592.57 & 326.68 & 354.08 & 933.94 \\
\hline$R^{*}(\mathrm{~kW})$ & 377.75 & 399.04 & 148.39 & 147.85 & 388.80 \\
\hline
\end{tabular}

(op-ex) peak power price. The peak shaving constraints in formulation, i.e., Constraint $P S$ are:

$$
\begin{array}{r}
0 \leq p_{t}+u_{t} \leq \max \left(p_{t}\right)-R, \forall t \in[1, T], \\
e_{0}=e_{T},
\end{array}
$$

where $p_{t}$ is the power curve before peak shaving, and $\max \left(p_{t}\right)$ is the original peak power. $u_{t}$ is the power change rate from the view of system level. $p_{t}+u_{t}$ is the new power curve after peak shaving, and $\max \left(p_{t}\right)-R$ is the new peak power. $e_{0}=e_{T}$ represents that energy stored in ESS is kept the same at the beginning and in the end of the time frame (in our study $T=1$ day). We formulate the optimization problem for ESS in peak shaving by putting Eq.(2)-(7) together with Eq. (15)-(16). The objective goal is to maximize the net profit and the decision variables are the same as those of RSR.

2) Case Study: We generate $p_{t}$ from a real HP workload trace collected from a data center that consists of 5,000 servers. The peak power of this trace is $1 \mathrm{MW}$, commonly seen in today's mid-size data center, and matches with the typical capacities of ESSs. Fig 3(a) is an example of $p_{t}$ in a day.

Unlike the optimal solution of RSR or contingency reserves that is either 0 or maximal capacity allowed (i.e., no feasible optimal solution), the optimal solution of peak shaving can be in between. Table II lists the optimal solutions of different ESSs for peak shaving of the power trace $p_{t}$ shown in Fig 3(a) All these optimal solutions lead to positive net profit. CAES has the maximal optimal net profit, though the corresponding capacities in the optimal solution is unrealistic due to its extremely small power and energy densities. LA and LI batteries have larger optimal net profit than UC and FW, though UC and FW can gain promising profit with very small capacities.

Fig 3(b) to 3(d) show the optimal net profit for varying energy and power capacities $\left(E_{c a p}, P_{c a p}\right)$ in peak shaving, for LI, UC and CAES, respectively. These contour plots present where the optimal solution for each ESS is located. Fig 3(b) also shows that LI batteries can gain profit from peak shaving in most cases, except when the power capacity is very small. In Fig 3(c), the profit of UC is larger than 0 only when both power and energy capacities are small, which shows that the marginal increase of the credit received from peak shaving by enlarging UC capacities is smaller than the increase in UC capacity cost. In Fig 3(d), CAES is always able to gain profit in peak shaving though large profit is not practical due to the limitations of power and energy densities.

Next, considering today's typical ESS capacities in peak shaving, the last row in Table III shows the optimal net profit and the corresponding optimal shaved power $R^{*}$ of ESSs with typical capacities in Table \ and under today's cap-ex and op-ex market prices. From the table, UC and FW fail to gain net profit, whereas LA, LI and CAES earn net profit around \$300-400 per day.
TABle III. COMPARING the Optimal Net Profit of Multiple TyPeS OF ESSS (WITH $E_{c a p}, P_{c a p}$ Listed IN TABLEI) IN PARTICIPATING DIFFERENT PROGRAMS.

\begin{tabular}{|c||c|c||c|c||c|c||c|c||c|c||}
\hline \multicolumn{1}{|c||}{} & \multicolumn{2}{c||}{ LA } & \multicolumn{2}{c||}{ LI } & \multicolumn{2}{c||}{ UC } & \multicolumn{2}{c||}{ FW } & \multicolumn{2}{c||}{ CAES } \\
\hline & Profit & $R^{*}$ & Profit & $R^{*}$ & Profit & $R^{*}$ & Profit & $R^{*}$ & Profit & $R^{*}$ \\
\hline RSR & $-16.4 \mathrm{k}$ & 0.17 & $-11.1 \mathrm{k}$ & 0.29 & $13.0 \mathrm{k}$ & 5.95 & $10.3 \mathrm{k}$ & 5.94 & $-0.3 \mathrm{k}$ & 0.004 \\
\hline CR & $-0.12 \mathrm{k}$ & 1.00 & $-0.10 \mathrm{k}$ & 1.00 & $-1.02 \mathrm{k}$ & 1.50 & $-0.85 \mathrm{k}$ & 1.49 & $-0.006 \mathrm{k}$ & 0.02 \\
\hline PS & $0.41 \mathrm{k}$ & 0.20 & $0.44 \mathrm{k}$ & 0.20 & $-0.46 \mathrm{k}$ & 0.21 & $-0.31 \mathrm{k}$ & 0.20 & $0.31 \mathrm{k}$ & 0.13 \\
\hline \multicolumn{8}{c}{$a_{\text {the unit of profit and } R^{*} \text { in table are \$/day and MW. }}$. CR: contingency reserve; PS: peak shaving. }
\end{tabular}

Fig $3(\mathrm{e})$ and Fig $3(\mathrm{f})$ presents the optimal net profit of peak shaving for multiple ESSs, via varying op-ex and cap-ex peak power prices, respectively. The black dashed lines show where the current market prices are around. Note that in Fig $3(\mathrm{e})$, the cap-ex price is fixed at $\$ 10 / \mathrm{W}$, while in Fig $3(\mathrm{f})$ the opex price is fixed at $\$ 12 / \mathrm{kW} /$ Month (both of them are current prices). Fig 3(e) illustrates that CAES, LI, and LA gain net profit (larger than 0) under most cases including the current situation, while UC and FW need much higher payment to gain net profit. Similar results hold for cap-ex price in Fig 3(f)

The peak shaving results presented here can be generalized to any scenario as long as its power trace has a similar pattern to Fig 3(a) This pattern is common in many scenarios [15], such as, weekday power consumption of offices, buildings and industries, power consumption of many types of data centers, e.g., data centers dealing with search workload (e.g., Google), communication workload (e.g., MSN), commercial and financial workload (e.g., stock exchange), etc.

\section{Discussion}

We provide the optimal net profit of each ESS technology across the programs in Table III for today's typical capacities and market reserve prices. From the table, LA, LI batteries and CAES gain profit from peak shaving, whereas UC and FW gain profit from RSR. None of them gain profit from contingency reserve, due to its low price and low calling frequency. The maximal profit earned from emerging RSR (by today's typical $\mathrm{UC}$ or FW) is up to 30 times of the maximal profit that can be earned from traditional peak shaving program (by LA or LI batteries), which shows that there is a great opportunity for an ESS to gain significant profit from RSR provision in today's ancillary market. For providing RSR, UC and FW are the best choices due to their extremely high tolerance for frequent charging/discharging, high efficiency and power density, and relatively low power capacity cost, while LA, LI batteries and CAES are better choices for peak shaving, or contingency reserves (though are not profitable), because of their relatively lower cost on energy capacity and lower self-discharge rate.

\section{MAnaging Participation in REgulation SERVICE RESERVES}

Given the potential profitability of ESS participation in RSR program, we now focus on the design of policies to enable this participation in practice. There are many challenges involved in such participation. For example, the provider is required to track an RSR signal that varies rapidly, bidirectionally, and is not known ahead of time. In addition, the revenue is deducted by tracking error, which creates a trade-off between reserve maximizing and signal tracking. In this section, we 


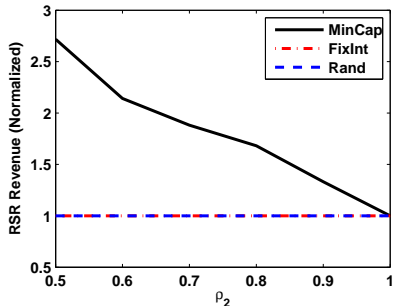

(a)

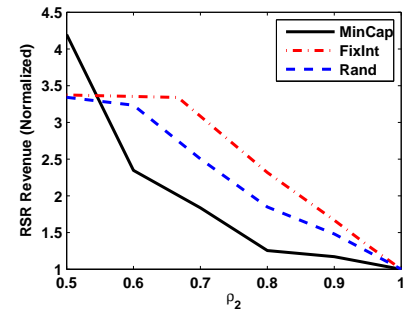

(b)
Fig. 4. The revenue of providing RSR via varying $\rho_{2}$, for LI batteries (in 4(a) and UC (in 4(b), with three heuristic offline solutions, respectively. The revenue is normalized to the value of $\rho_{2}=1$.

start by developing offline optimal solutions (assuming the RSR signal is known a priori), and then design practical online policies, in which the RSR signal is not known in advance.

\section{A. Offline policies for RSR}

In Section II-A, we introduce the offline optimal solution in the case when $\rho_{2}=1$ in Eq.91. $\rho_{2}=1$ simplifies the probabilistic constraint in Eq.96 to a deterministic constraint in Eq. 111. However, normally $\rho_{2}<1$ in practice, i.e., some violations of signal tracking are tolerable, which makes the optimization challenging

1) Policy overview: In this section, we propose three heuristic offline solutions to deal with the probabilistic constraint in Eq. (9) when $\rho_{2}<1$. The key idea behind these solutions is to determine when the signal should be tracked within the tolerance $\rho_{1}$ (i.e., satisfying Eq.(11)), and when the tolerance can be violated. Three solutions are as follows:

RandSelect: Randomly select $\rho_{2} T$ time intervals in $[1, T]$ to satisfy Eq. 111.

MinCapSelect: Select $\rho_{2} T$ time intervals in $[1, T]$ with smallest $\left|\beta_{t}\right|$ to satisfy Eq. 111. This design is based on the fact that tracking RSR signal at the time interval $t$ with larger $\left|\beta_{t}\right|$ requires larger power capacity.

FixIntSelect: Equally distribute $T-\rho_{2} T$ time intervals that are allowed to violate the Eq. 111) in $[1, T]$. This is for the purpose of enabling the policy to adjust amount of energy stored in ESSs without closely following the tracking constraint once a while.

2) Case study: Fig 4 shows the optimal RSR revenue solved based on Eq. (10) with three proposed offline methods via varying $\rho_{2}$, for LI batteries and UC with typical capacities listed in Table I] respectively. $\rho_{1}$ is fixed at 0.2 , as in Section III-A Note that since we use the typical capacities in all cases, the cost of ESS is fixed. Thus, it is equivalent to make comparisons of these three methods based on either the RSR revenue, i.e., Revenue $R S$ or the net profit originally used in the objective function of Eq. (10). In the figure, all the revenues are normalized by the revenue at $\rho_{2}=1$.

From Fig 4(a), MinCapSelect always achieves largest revenue for LI batteries when $\rho_{2}$ varies. The charge/discharge capacities, i.e., the power capacity are the main bottleneck for LI batteries to offer more reserves, while MinCapSelect can help reduce the requirement on power capacity by only tracking small $\left|\beta_{t}\right|$ and giving up tracking large $\left|\beta_{t}\right|$, hence enabling LI batteries to provide additional reserves. The results for UC, however, are different. The power capacity is no longer the bottleneck, as today's typical UC has a much stronger power capacity compared to its energy capacity. As a consequence, energy capacity turns out to be the bottleneck. In that case, MinCapSelect does not help, and is even worse than the random algorithm RandSelect. A solution that is able to utilize the limited energy capacity in a more efficient way can provide more reserves and earn higher revenue. FixIntSelect becomes a better solution shown in Fig 4(b), because it equally distributes time points where constraint violations are allowed across the whole time frame, so that the energy amount stored in ESS can be adjusted periodically and uniformly. Fig 4 also shows that the optimal revenue increases when $\rho_{2}$ decreases. Relaxing the signal tracking constraints by decreasing $\rho_{2}$ in general offers more flexibilities for ESSs to participate the RSR program, and therefore, enables them to gain larger profits.

\section{B. Online policies for RSR}

Prior offline solutions are based on the fact that RSR signal is known a priori, which is, however, not for the real case in practice. RSR signal is broadcast to demand side every few second in real time. In this section, we propose heuristic online ESS operational policies for RSR participation, where no information on the RSR signal is required in advance. In a practical scenario, the online policies handle the following problems: given the types and capacities of the ESS (i.e., assuming the ESS has been setup), how much reserve should be provided and how the ESS should be operated so that higher revenue from RSR participation can be gained and the feasibility of the participation can be guaranteed.

1) Policy overview: As discussed before, MinCapSelect provides the highest revenue for ESSs such as LI and LA batteries in the offline solution. Hence we design the online operational policies for LI and LA batteries based on the MinCapSelect solution, as follows:

Initialization: we calculate two thresholds $\theta_{0}$ and $\theta_{1}$, based on the requirement input $\left(\rho_{1}, \rho_{2}\right)$ from the market operator introduced before, and the historical data of RSR signal $\beta_{t}^{H}$, such that:

$$
\begin{array}{r}
\operatorname{Prob}\left\{\left|\beta_{t}^{H}\right| \leq \theta_{0}\right\}=\rho_{2}, \\
\theta_{1}=\left(1-\rho_{1}\right) \theta_{0} .
\end{array}
$$

Real-time Operation: at each time $t$, assuming the RSR signal value is $\beta_{t}^{R T}$, we determine the power rate $u_{t}$ by:

1) If $\left|\beta_{t}^{R T}\right|<\theta_{1}$ : we set $u_{t}=\beta_{t}^{R T}$, i.e., accurately track the signal;

2) If $\theta_{0} \geq\left|\beta_{t}^{R T}\right| \geq \theta_{1}$ : we set $u_{t}=\theta_{1} \operatorname{sign}\left(\beta_{t}^{R T}\right)$, i.e., cap the power rate $u_{t}$ at $\theta_{1}$;

3) If $\left|\beta_{t}^{R T}\right|>\theta_{0}$ : we no-longer track the signal, instead, we set $u_{t}$ to adjust the current energy stored $e_{t}$ back to a middle level $e_{m}=\frac{D o D * E_{c a p}}{2(1-\mu)}$ for future use (recall that $\mu$ is the self discharge rate);

4) Check and cap $u_{t}$ and $e_{t}$ based on power and energy capacity $\left(P_{c a p}, E_{c a p}\right)$ constraints of the ESS.

An advanced algorithm could be updating $\theta_{0}$ and $\theta_{1}$ adaptively and dynamically in real time based on tracking performance feedback.

For ESSs such as UC and FW, the FixIntSelect solution offers the highest revenue from the previous study of the offline solution. Therefore, we propose the online operational policy for UC and FW based on the FixIntSelect heuristic, as follows: 
Initialization: we calculate the intervals that adjust the stored energy in ESS based on the input $\rho_{2}: T_{i n t}=\left\lceil\frac{1}{1-\rho_{2}}\right\rceil$, i.e., we adjust the stored energy every $T_{\text {int }}$ period. In addition, we set $\theta_{1}=1-\rho_{1}$;

Real-time Operation: at each time $t$, assuming the RSR signal value is $\beta_{t}^{R T}$, we determine the power rate $u_{t}$ by:

1) Every $t=T_{\text {int }}$, we set $u_{t}$ to adjust the current energy stored $e_{t}$ back to middle level $e_{m}=\frac{D o D * E_{c a p}}{2(1-\mu)}$;

2) For $t \neq T_{\text {int }}$, if $\left|\beta_{t}^{R T}\right|<\theta_{1}$ : we set $u_{t}=\beta_{t}^{R T}$, i.e., accurately track the signal;

3) For $t \neq T_{\text {int }}$, if $\left|\beta_{t}^{R T}\right| \geq \theta_{1}$ : we set $u_{t}=$ $\theta_{1} \operatorname{sign}\left(\beta_{t}^{R T}\right)$, i.e., cap the power rate $u_{t}$ at $\theta_{1}$;

4) Check and cap $u_{t}$ and $e_{t}$ based on power and energy capacity $\left(P_{c a p}, E_{c a p}\right)$ constraints of the ESS.

Another essential issue in an online policy is the determination of the amount of reserve to provide, i.e. $R_{\text {onl }}$. Unlike the offline solution, in which the RSR signal is known ahead, thus an optimal $R$ can be calculated directly from the optimization formulation, the $R_{\text {onl }}$ for the online policies is required to be carefully estimated. We propose an approach to learn $R_{\text {onl }}$ from historical offline solutions, as $R_{\text {onl }}=\lambda R_{\text {min }}$, where $R_{\min }$ is the minimum of the offline optimal $R$ in the past 12 hours (the signal has been known in those hours, so offline optimal $R$ can be calculated), $\lambda$ is a discount value. We use $R_{\min }$ and select $\lambda$ to avoid aggressive estimation of $R_{o n l}$, and to guarantee feasibility of our policies. We select $\lambda=90 \%$ for LI batteries and $\lambda=75 \%$ for UC, because LI batteries have more stable results, much smaller provision and are less sensitive to variations of $\rho_{2}$ than UC shown in Section IV-A.

2) Case study: An aggressive claim of $R_{o n l}$ may lead to failure in reserve provisioning (i.e., constraints are violated) during the real-time operation, due to the limitations of ESS capacities. Hence, we first evaluate the feasibility of our online policies. We test the feasibility of our policies in the last 12 hours of a 1-day RSR signal. Each hour is a test case. In each test, we first calculate $R_{o n l}$ based on the offline optimal $R$ in previous 12 hours as proposed, and then simulate the online policies to check whether all constraints are satisfied during the test hour. We also evaluate the policies with different $\rho_{2}$. Our results show that these safely estimated $R_{\text {onl }}$ together with our policies satisfy all constraints and thus are feasible solutions in all test cases, for both LI batteries and UC.

Then we compare the RSR revenue of our online policies to the offline solutions in Fig 5 via varying $\rho_{2}$. For offline solutions, MinCapSelect is selected for LI batteries, and FixIntSelect is selected for UC, as they perform the best for LI batteries and UC respectively shown in Fig 4 and our online policies are designed based on them. All results in Fig 5 are normalized to the offline solution of $\rho_{2}=1$. From the figure, the proposed online solutions still receive promising revenues, though there is (as expected) a noticeable gap compared to offline solutions, due to the lack of RSR signal information, and the safe estimation of the reserve value $R_{\text {onl }}$. More importantly, however, the feasibility of such online policies is guaranteed with high confidence. There is the following tradeoff: an aggressive online policy may bring the revenue close to optimal offline solutions, while the real-time feasibility of such solution decreases at the same time.

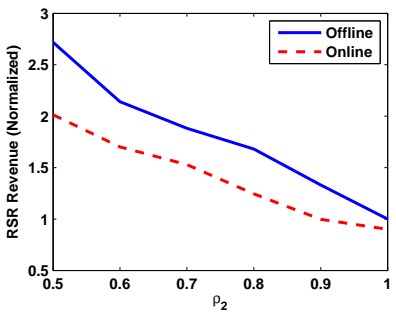

(a)

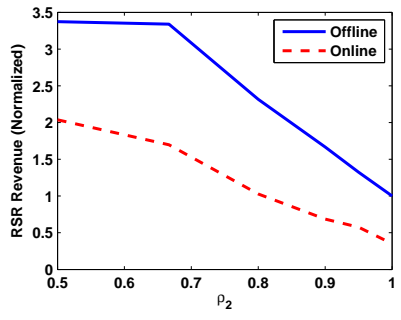

(b)
Fig. 5. The revenue of providing RSR via varying $\rho_{2}$, for LI batteries (in 5(a) and UC (in 5(b)], respectively, with offline and online solutions. The revenue is normalized to the value of $\rho_{2}=1$ in offline solutions.

\section{RELATED WORK}

Today's most popular ESSs include batteries, flywheels, ultra-capacitors and other emerging techniques, e.g., CAES, etc [19], [20]. These ESSs are modeled, for either ideal or non-ideal behaviors, and their system performance is evaluated [15], [21]. Recently, the hybrid electric energy storage system (HESS) is designed and investigated to enlarge the system storage capacity and improve the efficiency [22].

In tandem with the developments of ESSs, there is a growing attention on consumer (e.g., data centers, smart buildings and EVs) demand response and reserve provision in ancillary service markets. A few studies closely explore opportunities and challenges in demand response and ancillary service market for data centers to reduce cost [3], [4], [17], [23], [24]. Among them, RSR is especially of interest due to its high clearing price on reserves, and thus potentially large profits [18]. Other work proposes to jointly leverage a data center and Plug-in Hybrid EVs in regulation market to maximize the profit [5]. Some approaches co-schedule heating, ventilation, air conditioning (HVAC) and EVs for reducing the energy consumption and the peak energy demand [6].

ESSs are considered promising options for participation in power markets and demand response. A few previous studies propose control policies and evaluate the benefit of ESSs in real-time dynamic energy pricing programs [9], [25], peak shaving [15], [26], and frequency control [27], [28], respectively. However, most previous studies focus on traditional power market programs, though, ESSs are able to potentially receive higher profit from emerging ancillary service market, especially from RSR. In the space of RSR, some prior work surveys potential market chances and evaluates maturity of ESS participation in RSR [10], [11], [12], [13], but without formulating the detailed models of participation and evaluating the optimal solutions. The closest paper to the current work is [14]. However, [14] uses a simplified RSR participation model that does not consider the details of regulation accuracy constraints and penalties. Further, it assumes that the RSR signal always follows a statistical distribution known a priori, and without considering the reserve value and capacity planning for different ESSs. To the best of our knowledge, ours is the first paper to provide detailed models, evaluate and optimize the profits of various ESS technologies in not only traditional power market programs such as peak shaving, but also in emerging smart grid demand response such as RSR and contingency reserves, by proposing detailed reserve value and capacity planning, as well as online ESS operational policies. 


\section{CONCLUSION}

In this paper, we have modeled and studied the optimization solutions that maximize the net profit of various ESSs in different demand response programs. Our results show that typical UC and FW are the most profitable selections for RSR, while common battery techniques such as LI and LA batteries are the best choices for peak shaving. None of today's ESS technologies can earn positive net profits from merely providing contingency reserves. More importantly, applying UC/FW in RSR has the potential to be up to 30 times more profitable than LI/LA batteries for peak shaving. Additionally, we have proposed online policies for managing ESS participation in RSR program, the novel but most profitable option according to our studies. Our online policies guarantee the feasibility of RSR provisions, while also achieving significant profits.

\section{ACKNOWLEDGMENT}

This paper is supported by the NSF Grant 1464388 .

\section{REFERENCES}

[1] American Wind Energy Association: http://www.awea.org/Advocacy

[2] The 2020 climate and energy package. European Commission: http://ec.europa.eu/clima/policies/package

[3] Z. Liu, I. Liu, S. Low, and A. Wierman. Pricing data center demand response. In ACM international conference on measurement and modeling of computer systems, pages 111-123, 2014.

[4] H. Chen, M. C. Caramanis, and A. K. Coskun. Reducing the data center electricity costs through participation in smart grid programs. In IEEE International Green Computing Conference (IGCC), pages 1-10, 2014.

[5] M. Brocanelli, S. Li, X. Wang, and W. Zhang. Joint management of data centers and electric vehicles for maximized regulation profits. In IGCC, pages 1-10, 2013.

[6] T. Wei, Q. Zhu, and M. Maasoumy. Co-scheduling of HVAC control, EV charging and battery usage for building energy efficiency. In Intl. Conf. on Computer-Aided Design (ICCAD), pages 191-196, 2014.

[7] Enernoc. http://www.enernoc.com/

[8] Comverge. http://www.comverge.com/

[9] Y. Wang, X. Lin, M. Pedram, S. Park, and N. Chang. Optimal control of a grid-connected hybrid electrical energy storage system for homes. In Design, Automation and Test in Europe (DATE), pages 881-886, 2013.

[10] R. Walawalkar, J. Apt, and R. Mancini. Economics of electric energy storage for energy arbitrage and regulation in New York. Energy Policy, 35(4):2558-2568, 2007.

[11] K. Kumaraswamy and J. Cotrone. Evaluating the regulation market maturity for energy storage devices. The Electricity Journal, 26(10):7583, 2013.

[12] K. Vu, R. Masiello, and R. Fioravanti. Benefits of fast-response storage devices for system regulation in ISO markets. In IEEE Power and Energy Society General Meeting (PES), pages 1-8. IEEE, 2009.

[13] D. Fooladivanda, C. Rosenberg, and S. Garg. An analysis of energy storage and regulation. In IEEE Intl. Conf. on Smart Grid Communications (SmartGridComm), pages 91-96, 2014.

[14] Y. Kim, A. Raghunathan, and V. Raghunathan. Design and management of hybrid electrical energy storage systems for regulation services. In IGCC, pages 1-9, 2014.

[15] D. Wang, C. Ren, A. Sivasubramaniam, B. Urgaonkar, and H. Fathy. Energy storage in datacenters: what, where, and how much? ACM SIGMETRICS Performance Evaluation Review, 40(1):187-198, 2012.

[16] PJM (2013). market-based regulation [online]. http://pjm.com/markets-and-operations/ancillary-services

[17] D. Aikema, R. Simmonds, and H. Zareipour. Data centres in the ancillary services market. In IGCC, pages 1-10, 2012.

[18] H. Chen, M. C. Caramanis, and A. K. Coskun. The data center as a grid load stabilizer. In Design Automation Conference (ASP-DAC), IEEE 19th Asia and South Pacific, pages 105-112, 2014.
[19] S. McCluer and J. Christin. Comparing data center batteries, flywheels, and ultracapacitors. White Paper, 65, 2008.

[20] S. C. Smith, P. K. Sen, and B. Kroposki. Advancement of energy storage devices and applications in electrical power system. In IEEE PES, pages 1-8, 2008.

[21] Y. Ghiassi-Farrokhfal, S. Keshav, and C. Rosenberg. Toward a realistic performance analysis of storage systems in smart grids. Smart Grid, IEEE Transactions on, 6(1):402-410, 2015.

[22] M. Pedram, N. Chang, Y. Kim, and Y. Wang. Hybrid electrical energy storage systems. In Low-Power Electronics and Design (ISLPED), ACM/IEEE International Symposium on, pages 363-368, 2010.

[23] A. Wierman, Z. Liu, I. Liu, and H. Mohsenian-Rad. Opportunities and challenges for data center demand response. In $I G C C$, pages 1-10, 2014.

[24] Í. Goiri, M. E Haque, K. Le, R. Beauchea, T. D Nguyen, J. Guitart, J. Torres, and R. Bianchini. Matching renewable energy supply and demand in green datacenters. Ad Hoc Networks, 25:520-534, 2015.

[25] D. Zhu, Y. Wang, S. Yue, Q. Xie, M. Pedram, and N. Chang. Maximizing return on investment of a grid-connected hybrid electrical energy storage system. In ASP-DAC, pages 638-643, 2013.

[26] B. Aksanli, E. Pettis, and T. Rosing. Architecting efficient peak power shaving using batteries in data centers. In Modeling, Analysis and Simulation of Computer and Telecommunication Systems (MASCOTS), IEEE 21st International Symposium on, pages 242-253, 2013.

[27] A. Oudalov, D. Chartouni, and C. Ohler. Optimizing a battery energy storage system for primary frequency control. Power Systems, IEEE Transactions on, 22(3):1259-1266, Aug 2007.

[28] Y. Cho, J. W. Shim, S.-J. Kim, S. W. Min, and K. Hur. Enhanced frequency regulation service using hybrid energy storage system against increasing power-load variability. In PES, pages 1-5. IEEE, 2013. 